\title{
Kernos
}

Revue internationale et pluridisciplinaire de religion grecque antique

15 | 2002

Varia

\section{Annette HUPFLOHER, Kulte im kaiserzeitlichen Sparta. Eine Rekonstruktion anhand der Priesterämter}

\section{Olivier Gengler}

\section{(2) OpenEdition \\ Journals}

Édition électronique

URL : http://journals.openedition.org/kernos/1438

DOI : 10.4000/kernos. 1438

ISSN : 2034-7871

Éditeur

Centre international d'étude de la religion grecque antique

Édition imprimée

Date de publication : 1 janvier 2002

ISSN : 0776-3824

Référence électronique

Olivier Gengler, «Annette hupfoner, Kulte im kaiserzeitlichen Sparta. Eine Rekonstruktion anhand der Priesterämter », Kernos [En ligne], 15 | 2002, mis en ligne le 22 avril 2011, consulté le 21 septembre 2020. URL : http://journals.openedition.org/kernos/1438; DOI : https://doi.org/10.4000/kernos. 1438 
$\mathrm{rv}^{\mathrm{e}}$ siècle. Elle observe que nous assistons souvent, pendant cette période, à une "dionizzazione » ou à une " apollinizzazione » de divers sujets et termine son tour d'horizon avec Dionysos musicien. Ce ne sont pas les instruments musicaux qui déterminent le caractère d'une scène, mais le genre et le style de la musique qu'on y joue, qu'on ne peut reconnaître qu'à travers le contexte iconographique.

Cette recherche porte la marque de savants maîtres, bien visible dans la connaissance étendue et approfondie du matériel et dans l'attention prêtée au contexte dans le processus d'interprétation. On découvre souvent avec plaisir de belles observations de détail concernant des scènes musicales peu connues, autant de petites trouvailles qui ne sont pas reflétées dans les conclusions. C'est là une certaine injustice à l'égard de son sujet. Une vue d'ensemble aussi large comporte forcément peu d'éléments spectaculaires et semble manquer d'originalité. Mais en examinant les chapitres de plus près, on découvre une richesse d'observations, de remarques et de choix qui surprennent agréablement et ampli fient nos connaissances au sujet de la musique antique par l'examen des détails. Très utiles sont le classement des scènes dionysiaques, les observations sur les instruments à percussion, sur la syrinx, ainsi que sur certaines particularités iconographiques. Sur certains points, on peut signaler des lacunes bibliographiques, surtout en ce qui concerne le domaine de l'iconographie musicale dont une meilleure connaissance aurait sans doute contribué à rendre cette étude plus complète. Dans la discussion de sujets tels que le concours de Marsyas et d'Apollon (p. 35), celui de Thamyris et des Muses (p. 21), Orphée ou les femmes musiciennes (p. 72-73), l'A. semble ignorer une série d'articles publiés dans la revue spéciale d'iconographie musicale Imago Musicae, en particulier dans les volumes VII (1990) et VIII (1991) ou encore les actes du congrès de Hambourg, H. Heckmann et al. (éd.), Musikalische Ikonographie (Laaber Verlag, 1994). Dans la présentation du sujet, elle n'a pas pu éviter un certain nombre de redites (p. ex. le concours d'Apollon et de Marsyas, p. 35 et 55, 58). Sa lecture des images présente parfois des erreurs (elle ne reconnaît pas l'accordage, p. 41 et fig. 18) ou des interprétations trop littérales de certaines scènes (p. 22, la musique cadencée dans les processions des dieux), bien qu'elle reconnaisse pour la plupart correctement les conventions iconographiques. Un autre point difficile est son traitement un peu superficiel des Muses, des Nymphes et des Ménades comme divinités de la nature (p. 123-137). Toutefois ces remarques n'ont qu'une importance secondaire et ne diminuent pas l'intérêt de cette étude, dont les propositions ouvertes au dialogue et les découvertes conduisent à une meilleure compréhension du rôle de la musique dans la Grèce ancienne.

Ce livre présente un grand intérêt pour le musicologue, l'archéologue, l'historien, l'organologue et enfin pour tous ceux qui s'intéressent généralement à l'Antiquité classique.

Alexandra Goulaki Voutira (Département de Musique Université Aristote de Thessalonique)

Annette Hupfoher, Kulte im kaiserzeitlichen Sparta. Eine Rekonstruktion anband der Priesterämter, Berlin, Akademie Verlag, 2000. 1 vol. 17,5×24,5 $\mathrm{cm}, 245$ p. ISBN : 3-05-003548-X

Jusqu'il y a peu, l'histoire de Sparte à l'époque romaine n'avait guère suscité l'intérêt des chercheurs et était au mieux traitée comme l'ultime étape du déclin spartiate. Ainsi que le souligne l'A. en introduction, cette tendance s'observait également dans le domaine de l'histoire religieuse. En effet, dans leurs travaux fondamentaux, S. Wide (Lakonische Kulte, Leipzig, 1893) et L. Ziehen (art. "Sparta 
[Kulte]", ŘE 3 A2 [1929], c. 1453-1524) s'étaient efforcés de donner un aperçu aussi complet que possible des cultes spartiates, mais en privilégiant la recherche des formes primitives au détriment des états les plus récents, considérés comme autant d'affadissements du modèle dorien original. En outre, ils escomptaient atteindre cet état originel à travers des sources de toutes époques et de toutes natures amalgamées en un ensemble intemporel. Or, précisément, les sources les plus substantielles pour l'étude de la religion spartiate datent de l'époque impériale romaine et il est bien évident que les phénomènes religieux sont tout aussi sensibles à l'évolution que les autres phénomènes historiques.

Convaincue de l'interdépendance entre la structure sociale de la cité et l'organisation de ses cultes, l'A. en appelle donc à une approche qui prenne en compte le contexte dans lequel se déployaient ces cultes et qui mette en évidence l'importance relative de chacun dans le système religieux. L'A. entend donc donner un aperçu du panthéon spartiate tel qu'il a été honoré à un moment donné de l'histoire. L'état de la documentation l'a incitée à se tourner vers la période impériale romaine qui, comme nous l'avons dit, nous est connue par les sources les plus riches en matière d'histoire religieuse: les inscriptions et la Périégèse de Pausanias.

L'œuvre de Pausanias est cependant ambiguë, car nullement destinée à offrir un aperçu fidèle des réalités de son époque. L'A. lui a donc préféré l'éclairage direct, quoique partiel, des sources épigraphiques, s'appuyant sur la Périégèse à titre de confirmation. Les sources archéologiques, qui auraient été de nature à éclairer également l'étude des cultes, ne sont cependant disponibles que pour un nombre insuffisant de sanctuaires en l'état actuel des recherches. Les inscriptions d'époque impériale ont en revanche bénéficié des recherches récentes menées par A. Spawforth et N. Kennell. L'A. a d'ailleurs suivi les traces des deux chercheurs qui avaient montré l'intérêt de ces documents pour l'histoire des cultes, le premier en proposant voici une dizaine d'années une brève étude de la religion spartiate à l'époque romaine ("Spartan Cults under the Roman Empire: Some Notes", in J.M. Sanders, Pbilolakon, Londres, 1992, p. 227-238) et le second en prenant l'époque romaine pour point de départ dans sa tentative de reconstruction du système éducatif spartiate (The Gymnasium of Virtue, Chapel Hill, 1995).

C'est donc la documentation épigraphique qui est ici privilégiée et particulièrement les inscriptions honorifiques pour des prêtres ou des prêtresses. L'A. escompte ainsi révéler le panthéon en activité à l'époque, ce que ne permet pas le seul texte de Pausanias, et développer une analyse sociologique du clergé spartiate, mettant en évidence les particularités relatives à l'appartenance sociale, à l'origine familiale ou au sexe du personnel du culte.

Le plan adopté se veut le reflet exact du double objectif de la recherche : proposer un aperçu des cultes en activité à l'époque impériale à travers une étude du personnel investi dans l'organisation de ces cultes. Les différentes divinités honorées sont donc traitées systématiquement au sein de trois grandes parties consacrées respectivement aux charges de prêtre occupées par des femmes, à celles occupées par des femmes et/ou des hommes, et à celles occupées par des hommes. Pour chaque partie, un cas particulier est analysé en détail.

L'étude porte sur soixante personnes ayant exercé une trentaine de fonctions cultuelles entre le milieu du $\mathrm{I}^{\text {er }}$ et le milieu du III $^{\mathrm{e}}$ siècle après J.-C., période sur laquelle se distribuent les témoignages épigraphiques connus. D'emblée, il faut souligner l'étroitesse du corpus étudié. Malgré les tentatives de synthèse proposées en fin de volume, il faut reconnaître que le trop petit nombre de documents ne permet pas de dégager des tendances claires ni de répondre de manière satisfaisante aux questions annoncées en introduction. 
Dans la perspective de l'A., l'analyse des prêtrises devait mettre en évidence les cultes en activité. Son objectif était de pouvoir déterminer quels cultes étaient effectivement au cœur de la vie civique à l'époque impériale. Dénombrant les prêtres attestés pour chaque divinité, elle a ainsi noté la prééminence du culte impérial, associé au culte de Zeus, puis du culte des Dioscures et de Déméter à l'Éleusinion du Taygète. Devant la discrétion, dans les textes étudiés, d'une divinité comme Artémis Orthia, dont le culte à l'époque impériale est pourtant attesté archéologiquement et dans les sources littéraires, l'A. est amenée à nuancer quelque peu la portée de ses conclusions. En effet, même lorsque l'on possède un matériel épigraphique important en provenance d'un sanctuaire, comme c'est le cas pour celui d'Orthia, il est possible que son clergé échappe à l'analyse. Selon nous, la nuance est importante car elle limite la possibilité de donner un reflet du panthéon en activité à travers les seules prêtrises, a fortiori sur base de dénombrements dépendants de l'état de la documentation et de la nature des sources. En outre, alors que la comparaison entre les données des inscriptions et du texte de Pausanias tend à confirmer l'information de celui-ci, les cultes attestés dans les inscriptions ne représentent qu'un tiers des lieux de cultes divins répertoriés par le Périégète; or, comme le montre l'exemple d'Orthia, tous n'étaient pas tombés en désuétude à son époque.

Malgré cette limite, le travail est de qualité et apporte un éclairage nouveau sur les cultes spartiates et sur la manière de les étudier. L'approche très respectueuse des sources offre des cultes connus par les inscriptions d'époque impé riale une vision cohérente et solide qui ne demande qu'à être étayée et amplifiée. Au niveau de la présentation, on regrettera que l'A. n'ait pas muni son étude d'un index des textes étudiés qui était pourtant nécessaire dans une étude fondée essentiellement sur les inscriptions. À côté de quelques coquilles, particulièrement dans les titres de travaux en français, et plusieurs erreurs dans la composition du grec, il convient d'apporter les précisions suivantes: p. 149, l'inscription du Musée de Sparte $n^{\circ} 6859$ présentée comme inédite porte en réalité le $\mathrm{n}^{\circ} 6589$ et a été publiée par G. Steinhauer dans $A B S A 93$ (1998), p. 435-438 n 5; p. 188, 201

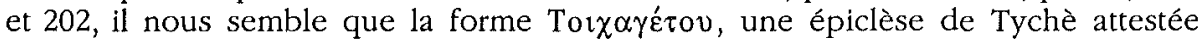

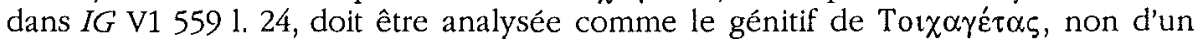
hypothétique Toichagetos.

O. Gengler
(FNRS - Faculté Univ, Notre-Dame de la Paix, Namur)

Geneviève Hoffmann (éd.), Les pierres de l'offrande. Autour de l'œuvre de Christoph W. Clairmont. Actes édités avec la collaboration d'Adrienne LezziHafter, Zürich, Akanthus, 2001. 1 vol. $17 \times 24,5 \mathrm{~cm}, 208$ p., nbr. ill. ISBN : 3905083-15-9.

Ces pierres de l'offrande sont les stèles funéraires, qui ont été l'objet des soins de Chr. W. Clairmont pendant de nombreuses années et continuent de l'être. C'est donc en l'honneur de ce savant que s'est tenu, en décembre 1998, un colloque à Clermont-Ferrand. Après une présentation de l'éditrice et une intervention en forme de bilan historiographique de la part du jubilaire, l'ouvrage, très soigneusement édité, s'articule en quatre parties : Archéologie grecque, Iconographie et société, Religion et droit, Muséographie. Ce sont évidemment les troisième et quatrième sections qui intéressent plus directement les lecteurs de Kernos ( $c f$. le détail du sommaire infra, p. 545-546). J'y épinglerai plusieurs points. Tout d'abord, les analyses de V. Dasen sur la gémellité, qui mettent bien évidence les questions de désordre, de souillure et d'illégitimité que soulève le caractère excessif des naissances multiples. Ensuite celles de Michèle Daumas qui a repris, 